\title{
Boundary value problem for nonlinear fractional differential equations of variable order via Kuratowski MNC technique
}

\author{
Amar Benkerrouche ${ }^{1}$, Dumitru Baleanu ${ }^{2,3}$, Mohammed Said Souid ${ }^{4}$, Ali Hakem ${ }^{1}$ and Mustafa Inc I,6,7 $^{*}$ (D)
}

Correspondence: minc@firat.edu.tr

${ }^{5}$ Department of Computer Engineering, Biruni University,

Istanbul, Turkey

${ }^{6}$ Science Faculty, Department of Mathematics, Firat University, 23119

Elazig, Turkey

Full list of author information is

available at the end of the article

\section{Springer}

\begin{abstract}
In the present research study, for a given multiterm boundary value problem (BVP) involving the Riemann-Liouville fractional differential equation of variable order, the existence properties are analyzed. To achieve this aim, we firstly investigate some specifications of this kind of variable-order operators, and then we derive the required criteria to confirm the existence of solution and study the stability of the obtained solution in the sense of Ulam-Hyers-Rassias (UHR). All results in this study are established with the help of the Darbo's fixed point theorem (DFPT) combined with Kuratowski measure of noncompactness (KMNC). We construct an example to illustrate the validity of our observed results.
\end{abstract}

MSC: 26A33; 34K37

Keywords: Fractional differential equations of variable order; Boundary value problem; Darbo's fixed point theorem; Measure of noncompactness;

Ulam-Hyers-Rassias stability

\section{Introduction}

The idea of fractional calculus is replacing the natural numbers in the derivative order with rational ones. Although it seems an elementary consideration, it has an interesting correspondence in explaining some physical phenomena. In the last two decades, significant research studies appeared on this topic, and some papers dealt with the existence of solutions to the problems of variable order; see, for example, [1-7].

Whereas many researchers investigated the existence of solutions for fractional constantorder problems, the existence of solutions of variable-order problems is rarely mentioned in the literature (we refer to [8-13]).

As a result of our investigation in this interesting research field, our findings are unique and noteworthy.

Furthermore, all of the findings in this paper have a great potential to be applied in a variety of transdisciplinary science applications. With the support of our original findings in this research study, we are able to do further research on this open research topic. In

(c) The Author(s) 2021. This article is licensed under a Creative Commons Attribution 4.0 International License, which permits use sharing, adaptation, distribution and reproduction in any medium or format, as long as you give appropriate credit to the original author(s) and the source, provide a link to the Creative Commons licence, and indicate if changes were made. The images or other third party material in this article are included in the article's Creative Commons licence, unless indicated otherwise in a credit line to the material. If material is not included in the article's Creative Commons licence and your intended use is not permitted by statutory regulation or exceeds the permitted use, you will need to obtain permission directly from the copyright holder. To view a copy of this licence, visit http://creativecommons.org/licenses/by/4.0/ 
other words, the proposed BVP can be extended to more sophisticated real mathematical fractional models in the future.

In particular, Bai et al. [14] studied the following problem:

$$
\left\{\begin{array}{l}
\left.\left.{ }^{c} D_{0^{+}}^{u} x(t)=f\left(t, x(t), I_{0^{+}}^{u} x(t)\right), \quad t \in J:=[a, b], u \in\right] 0,1\right], \\
x(a)=x_{a}
\end{array}\right.
$$

where ${ }^{c} D_{0^{+}}^{u}$ and $I_{0^{+}}^{u}$ stand for the Caputo-Hadamard derivative and Hadamard integral operators of order $u$, respectively, $f$ is a given function, $x_{a} \in \mathbb{R}$, and $0<a<b<\infty$.

Inspired by [14] and [1-5], we deal with the boundary value problem (BVP)

$$
\left\{\begin{array}{l}
D_{0^{+}}^{u(t)} x(t)=f_{1}\left(t, x(t), I_{0^{+}}^{u(t)} x(t)\right), \quad t \in J:=[0, T], \\
x(0)=0, \quad x(T)=0,
\end{array}\right.
$$

where $1<u(t) \leq 2, f_{1}: J \times X \times X \rightarrow X$ is a continuous function, and $D_{0^{+}}^{u(t)}$ and $I_{0^{+}}^{u(t)}$ are the Riemann-Liouville fractional derivative and integral of variable order $u(t)$.

In this paper, we investigate the solution of (1). Further, we study the stability of the obtained solution of (1) in the Ulam-Hyers-Rassias (UHR) sense.

\section{Preliminaries}

In this section, we introduce some important fundamental definitions that will be needed for obtaining our results in the next sections.

By $C(J, X)$ we denote the Banach space of continuous functions $\varkappa: J \rightarrow X$ with the norm

$$
\|\varkappa\|=\sup \{\|\varkappa(t)\|: t \in J\}
$$

where $X$ is a real (or complex) Banach space.

For $-\infty<a_{1}<a_{2}<+\infty$, we consider the mappings $u(t):\left[a_{1}, a_{2}\right] \rightarrow(0,+\infty)$ and $v(t)$ : $\left[a_{1}, a_{2}\right] \rightarrow(n-1, n)$. Then the left Riemann-Liouville fractional integral (RLFI) of variable order $u(t)$ for function $h_{1}(t)$ is [15-17]

$$
I_{a_{1}^{+}}^{u(t)} h_{1}(t)=\int_{a_{1}}^{t} \frac{(t-s)^{u(t)-1}}{\Gamma(u(t))} h_{1}(s) d s, \quad t>a_{1}
$$

and the left Riemann-Liouville fractional derivative (RLFD) of variable-order $v(t)$ for function $h_{1}(t)$ is $[15-17]$

$$
D_{a_{1}^{+}}^{\nu(t)} h_{1}(t)=\left(\frac{d}{d t}\right)^{n} I_{a_{1}^{+}}^{n-v(t)} h_{1}(t)=\left(\frac{d}{d t}\right)^{n} \int_{a_{1}}^{t} \frac{(t-s)^{n-v(t)-1}}{\Gamma(n-v(t))} h_{1}(s) d s, \quad t>a_{1} .
$$

In case of constant $u(t)$ and $v(t)$, RLFI and RLFD coincide with the standard RiemannLiouville fractional derivative and integral, respectively; see, for example, [15, 16, 18].

Let us recall the following pivotal observation.

Lemma 2.1 ([18]) Let $\alpha_{1}, \alpha_{2}>0, a_{1}>0, h_{1} \in L\left(a_{1}, a_{2}\right)$, and $D_{a_{1}^{+}}^{\alpha_{1}} h_{1} \in L\left(a_{1}, a_{2}\right)$. Then the differential equation

$$
D_{a_{1}^{+}}^{\alpha_{1}} h_{1}=0
$$


has the unique solution

$$
h_{1}(t)=\omega_{1}\left(t-a_{1}\right)^{\alpha_{1}-1}+\omega_{2}\left(t-a_{1}\right)^{\alpha_{1}-2}+\cdots+\omega_{n}\left(t-a_{1}\right)^{\alpha_{1}-n},
$$

and

$$
I_{a_{1}^{+}}^{\alpha_{1}} D_{a_{1}^{+}}^{\alpha_{1}} h_{1}(t)=h_{1}(t)+\omega_{1}\left(t-a_{1}\right)^{\alpha_{1}-1}+\omega_{2}\left(t-a_{1}\right)^{\alpha_{1}-2}+\cdots+\omega_{n}\left(t-a_{1}\right)^{\alpha_{1}-n}
$$

with $n-1<\alpha_{1} \leq n, \omega_{\ell} \in \mathbb{R}, \ell=1,2, \ldots, n$.

Furthermore,

$$
D_{a_{1}^{+}}^{\alpha_{1}} I_{a_{1}^{+}}^{\alpha_{1}} h_{1}(t)=h_{1}(t)
$$

and

$$
I_{a_{1}^{+}}^{\alpha_{1}} I_{a_{1}^{+}}^{\alpha_{2}} h_{1}(t)=I_{a_{1}^{+}}^{\alpha_{2}} I_{a_{1}^{+}}^{\alpha_{1}} h_{1}(t)=I_{a_{1}^{+}}^{\alpha_{1}+\alpha_{2}} h_{1}(t)
$$

Remark 2.1 ([19-21]) Note that the semigroup property is not fulfilled for general functions $u(t), v(t)$, that is,

$$
I_{a_{1}^{+}}^{u(t)} I_{a_{1}^{+}}^{v(t)} h_{1}(t) \neq I_{a_{1}^{+}}^{u(t)+v(t)} h_{1}(t)
$$

Example 2.1 Let

$$
\begin{aligned}
u(t)=t, \quad t & \in[0,4], \quad v(t)=\left\{\begin{array}{ll}
2, & t \in[0,1], \\
3, & t \in] 1,4],
\end{array} h_{1}(t)=2, \quad t \in[0,4],\right. \\
I_{0^{+}}^{u(t)} I_{0^{+}}^{v(t)} h_{1}(t) & =\int_{0}^{t} \frac{(t-s)^{u(t)-1}}{\Gamma(u(t))} \int_{0}^{s} \frac{(s-\tau)^{v(s)-1}}{\Gamma(v(s))} h_{1}(\tau) d \tau d s \\
& =\int_{0}^{t} \frac{(t-s)^{t-1}}{\Gamma(t)}\left[\int_{0}^{1} \frac{(s-\tau)}{\Gamma(2)} 2 d \tau+\int_{1}^{s} \frac{(s-\tau)^{2}}{\Gamma(3)} 2 d \tau\right] d s \\
& =\int_{0}^{t} \frac{(t-s)^{t-1}}{\Gamma(t)}\left[2 s-1+\frac{(s-1)^{3}}{3}\right] d s,
\end{aligned}
$$

and

$$
I_{0^{+}}^{u(t)+v(t)} h_{1}(t) \mid=\int_{0}^{t} \frac{(t-s)^{u(t)+v(t)-1}}{\Gamma(u(t)+v(t))} h_{1}(s) d s .
$$

So we get

$$
\left.I_{0^{+}}^{u(t)} I_{0^{+}}^{\nu(t)} h_{1}(t)\right|_{t=3}=\int_{0}^{3} \frac{(3-s)^{2}}{\Gamma(3)}\left[2 s-1+\frac{(s-1)^{3}}{3}\right] d s=\frac{21}{10},
$$




$$
\begin{aligned}
\left.I_{0^{+}}^{u(t)+v(t)} h_{1}(t)\right|_{t=3}= & \int_{0}^{3} \frac{(3-s)^{u(t)+v(t)-1}}{\Gamma(u(t)+v(t))} h_{1}(s) d s \\
= & \int_{0}^{1} \frac{(3-s)^{4}}{\Gamma(5)} 2 d s+\int_{1}^{3} \frac{(3-s)^{5}}{\Gamma(6)} 2 d s \\
= & \frac{1}{12} \int_{0}^{1}\left(s^{4}-12 s^{3}+54 s^{2}-108 s+81\right) d s \\
& +\frac{1}{60} \int_{1}^{3}\left(-s^{5}+15 s^{4}-90 s^{3}+270 s^{2}-405 s+243\right) d s \\
= & \frac{665}{180} .
\end{aligned}
$$

Therefore we obtain

$$
\left.I_{0^{+}}^{u(t)} I_{0^{+}}^{\nu(t)} h_{1}(t)\right|_{t=3} \neq\left. I_{0^{+}}^{u(t)+v(t)} h_{1}(t)\right|_{t=3} .
$$

Lemma 2.2 ([22]) Let $u: J \rightarrow(1,2]$ be a continuous function. Then for

$$
h_{1} \in C_{\delta}(J, X)=\left\{h_{1}(t) \in C(J, X), t^{\delta} h_{1}(t) \in C(J, X)\right\} \quad\left(0 \leq \delta \leq \min _{t \in J}|u(t)|\right),
$$

the variable-order fractional integral $I_{0^{+}}^{u(t)} h_{1}(t)$ exists for any points on $J$.

Lemma 2.3 ([22]) Let $u: J \rightarrow(1,2]$ be a continuous function. Then

$$
I_{0^{+}}^{u(t)} h_{1}(t) \in C(J, X) \quad \text { for } h_{1} \in C(J, X)
$$

Definition 2.1 ([23-25]) A set $I \subset \mathbb{R}$ is called a generalized interval if it is either an interval, or $\left\{a_{1}\right\}$, or \{\} .

A finite set $\mathcal{P}$ of generalized intervals is called a partition of $I$ if each $x \in I$ lies in exactly one generalized interval $E$ in $\mathcal{P}$.

A function $g: I \rightarrow X$ is called piecewise constant with respect to partition $\mathcal{P}$ of $I$ if for any $E \in \mathcal{P}, g$ is constant on $E$.

\subsection{Measure of noncompactness}

In this subsection, we discuss some necessary background information about KMNCs.

Definition 2.2 ([26]) Let $X$ be a Banach space, and let $\Omega_{X}$ be the bounded subsets of $X$. A KMNC is a mapping $\zeta: \Omega_{X} \rightarrow[0, \infty]$ constructed as follows:

$$
\zeta(D)=\inf \left\{\epsilon>0: D\left(\in \Omega_{X}\right) \subseteq \cup_{\ell=1}^{n} D_{\ell}, \operatorname{diam}\left(D_{\ell}\right) \leq \epsilon\right\}
$$

where

$$
\operatorname{diam}\left(D_{\ell}\right)=\sup \left\{\|x-y\|: x, y \in D_{\ell}\right\}
$$

The following properties are valid for KMNCs. 
Proposition $2.1([26,27])$ Let $X$ be a Banach space, and let $D, D_{1}$, and $D_{2}$ be bounded subsets of $X$. Then:

1. $\zeta(D)=0 \Longleftrightarrow D$ is relatively compact.

2. $\zeta(\phi)=0$.

3. $\zeta(D)=\zeta(\bar{D})=\zeta(\operatorname{conv} D)$.

4. $D_{1} \subset D_{2} \Longrightarrow \zeta\left(D_{1}\right) \leq \zeta\left(D_{2}\right)$.

5. $\zeta\left(D_{1}+D_{2}\right) \leq \zeta\left(D_{1}\right)+\zeta\left(D_{2}\right)$

6. $\zeta(\lambda D)=|\lambda| \zeta(D), \lambda \in \mathbb{R}$.

7. $\zeta\left(D_{1} \cup D_{2}\right)=\operatorname{Max}\left\{\zeta\left(D_{1}\right), \zeta\left(D_{2}\right)\right\}$.

8. $\zeta\left(D_{1} \cap D_{2}\right)=\operatorname{Min}\left\{\zeta\left(D_{1}\right), \zeta\left(D_{2}\right)\right\}$.

9. $\zeta\left(D+x_{0}\right)=\zeta(D)$ for all $x_{0} \in X$.

Lemma 2.4 ([28]) If $U \subset C(J, X)$ is an equicontinuous and bounded set, then:

(i) the function $\zeta(U(t))$ is continuous for $t \in J$, and

$$
\widehat{\zeta}(U)=\sup _{t \in J} \zeta(U(t))
$$

(ii) $\zeta\left(\int_{0}^{T} x(\theta) d \theta: x \in U\right) \leq \int_{0}^{T} \zeta(U(\theta)) d \theta$,

where

$$
U(s)=\{x(s): x \in U\}, \quad s \in J .
$$

Theorem 2.1 (DFPT [26]) Let $\Lambda$ be nonempty, closed, bounded, and convex subset of a Banach space $X$, and let $\digamma: \Lambda \longrightarrow \Lambda$ be a continuous operator satisfying

$$
\zeta(\digamma(S)) \leq k \zeta(S) \quad \text { for any }(S \neq \emptyset) \subset \Lambda, k \in[0,1),
$$

that is, $F$ is a $k$-set contraction.

Then $F$ has at least one fixed point in $\Lambda$.

Definition 2.3 ([29]) Let $\vartheta \in C(J, X)$. Equation of (1) is UHR stable with respect to $\vartheta$ if there exists $c_{f}>0$ such that for any $\epsilon>0$ and every solution $z \in C(J, X)$ of the inequality

$$
\left\|D_{0^{+}}^{u(t)} z(t)-f\left(t, z(t), I_{0^{+}}^{u(t)} z(t)\right)\right\| \leq \epsilon \vartheta(t), \quad t \in J,
$$

there exists a solution $x \in C(J, X)$ of equation (1) with

$$
\|z(t)-x(t)\| \leq c_{f} \in \vartheta(t), \quad t \in J .
$$

\section{Existence of solutions}

Let us introduce the following assumptions:

(H1) Let $n \in \mathbb{N}$ be an integer, let $\mathcal{P}=\left\{J_{1}:=\left[0, T_{1}\right], J_{2}:=\left(T_{1}, T_{2}\right], J_{3}:=\left(T_{2}, T_{3}\right], \ldots, J_{n}:=\left(T_{n-1}, T\right]\right\}$ be a partition of the interval $J$, and let $u(t): J \rightarrow(1,2]$ be a piecewise constant function with respect to 
$\mathcal{P}$, that is,

$$
u(t)=\sum_{\ell=1}^{n} u_{\ell} I_{\ell}(t)= \begin{cases}u_{1} & \text { for } t \in J_{1} \\ u_{2} & \text { for } t \in J_{2} \\ \vdots & \\ u_{n} & \text { for } t \in J_{n}\end{cases}
$$

where $1<u_{\ell} \leq 2$ are constants, and $I_{\ell}$ is the indicator of the interval $J_{\ell}:=\left(T_{\ell-1}, T_{\ell}\right], \ell=1,2, \ldots, n$ (with $T_{0}=0, T_{n}=T$ ), such that

$$
I_{\ell}(t)= \begin{cases}1 & \text { for } t \in J_{\ell} \\ 0 & \text { elsewhere }\end{cases}
$$

(H2) Let $t^{\delta} f_{1}: J \times X \times X \rightarrow X$ be a continuous function $\left(0 \leq \delta \leq \min _{t \in J}|(u(t))|\right)$. There exist constants $K, L>0$ such that

$$
t^{\delta}\left\|f_{1}\left(t, y_{1}, z_{1}\right)-f_{1}\left(t, y_{2}, z_{2}\right)\right\| \leq K\left\|y_{1}-y_{2}\right\|+L\left\|z_{1}-z_{2}\right\| \quad \text { for all } y_{1}, y_{2}, z_{1}, z_{2} \in X \text { and } t \in J \text {. }
$$

Remark 3.1 According to the remark of [30] on page 20, we can easily show that condition $(\mathrm{H} 2)$ and the inequality

$$
\zeta\left(t^{\delta}\left\|f_{1}\left(t, B_{1}, B_{2}\right)\right\|\right) \leq K \zeta\left(B_{1}\right)+L \zeta\left(B_{2}\right)
$$

are equivalent for any bounded sets $B_{1}, B_{2} \subset X$ and $t \in J$.

Further, for a given set $U$ of functions $u: J \rightarrow X$, let us denote

$$
U(t)=\{u(t), u \in U\}, \quad t \in J
$$

and

$$
U(J)=\{U(t): v \in U, t \in J\}
$$

Let us now prove the existence of solution for the BVP (1) via the concepts of MNCK and DFPT.

For $\ell \in\{1,2, \ldots, n\}$, by $E_{\ell}=C\left(J_{\ell}, X\right)$ we denote the Banach space of continuous functions $x: J_{\ell} \rightarrow X$ equipped with the norm

$$
\|x\|_{E_{\ell}}=\sup _{t \in J_{\ell}}\|x(t)\|
$$

First, we analyze BVP (1).

By (3) the equation of BVP (1) can be expressed as

$$
\frac{d^{2}}{d t^{2}} \int_{0}^{t} \frac{(t-s)^{1-u(t)}}{\Gamma(2-u(t))} x(s) d s=f_{1}\left(t, x(t), I_{0^{+}}^{u(t)} x(t)\right), \quad t \in J
$$


Taking (H1) into account, equation(4) in the interval $J_{\ell}, \ell=1,2, \ldots, n$, can be written as

$$
\begin{aligned}
& \frac{d^{2}}{d t^{2}}\left(\int_{0}^{T_{1}} \frac{(t-s)^{1-u_{1}}}{\Gamma\left(2-u_{1}\right)} x(s) d s+\cdots+\int_{T_{\ell-1}}^{t} \frac{(t-s)^{1-u_{\ell}}}{\Gamma\left(2-u_{\ell}\right)} x(s) d s\right) \\
& \quad=f_{1}\left(t, x(t), I_{0^{+}}^{u_{\ell}} x(t)\right), \quad t \in J_{\ell} .
\end{aligned}
$$

Now we introduce the solution to BVP (1).

Definition 3.1 BVP (1) has a solution if there are functions $x_{\ell}, \ell=1,2, \ldots, n$, such that $x_{\ell} \in C\left(\left[0, T_{\ell}\right], X\right)$ fulfills equation $(5)$ and $x_{\ell}(0)=0=x_{\ell}\left(T_{\ell}\right)$.

According the above observation, BVP (1) can be expressed for any $t \in J_{l}, l=1,2, \ldots, n$, as (5).

For $0 \leq t \leq T_{\ell-1}$, taking $x(t) \equiv 0$, we can write (5) as

$$
D_{T_{\ell-1}^{+}}^{u_{\ell}} x(t)=f_{1}\left(t, x(t), I_{T_{\ell-1}^{+}}^{u_{\ell}} x(t)\right), \quad t \in J_{\ell} .
$$

We will deal with the following BVP:

$$
\left\{\begin{array}{l}
D_{T_{\ell-1}^{+}}^{u_{\ell}} x(t)=f_{1}\left(t, x(t), I_{T_{\ell-1}^{+}}^{u_{\ell}} x(t)\right), \quad t \in J_{\ell}, \\
x\left(T_{\ell-1}\right)=0, \quad x\left(T_{\ell}\right)=0 .
\end{array}\right.
$$

For our purpose, the following lemma will be the basis of the solution of (6).

Lemma 3.1 A function $x \in E_{\ell}$ forms a solution of (6) if and only if $x$ fulfills the integral equation

$$
\begin{aligned}
x(t)= & -\left(T_{\ell}-T_{\ell-1}\right)^{1-u_{\ell}}\left(t-T_{\ell-1}\right)^{u_{\ell}-1} I_{T_{\ell-1}^{+}}^{u_{\ell}} f_{1}\left(T_{\ell}, x\left(T_{\ell}\right), I_{T_{\ell-1}^{+}}^{u_{\ell}} x\left(T_{\ell}\right)\right) \\
& +I_{T_{\ell-1}^{+}}^{u_{\ell}} f_{1}\left(t, x(t), I_{T_{\ell-1}^{+}}^{u_{\ell}} x(t)\right) .
\end{aligned}
$$

Proof Let $x \in E_{\ell}$ be solution of problem (6). Applying the operator $I_{T_{\ell-1}^{+}}^{u_{\ell}}$ to both sides of (6), from Lemma 2.1 we find

$$
\begin{aligned}
x(t)= & \omega_{1}\left(t-T_{\ell-1}\right)^{u_{\ell}-1}+\omega_{2}\left(t-T_{\ell-1}\right)^{u_{\ell}-2} \\
& +\frac{1}{\Gamma\left(u_{\ell}\right)} \int_{T_{\ell-1}}^{t}(t-s)^{u_{\ell}-1} f_{1}\left(s, x(s), I_{T_{\ell-1}^{+}}^{u_{\ell}} x(s)\right) d s, \quad t \in J_{\ell} .
\end{aligned}
$$

Due to the assumption on the function $f_{1}$ along with $x\left(T_{\ell-1}\right)=0$, we conclude that $\omega_{2}=0$.

Let $x$ satisfy $x\left(T_{\ell}\right)=0$. Observe that

$$
\omega_{1}=-\left(T_{\ell}-T_{\ell-1}\right)^{1-u_{\ell}} I_{T_{\ell-1}^{+}}^{u_{\ell}} f_{1}\left(T_{\ell}, x\left(T_{\ell}\right), I_{T_{\ell-1}^{+}}^{u_{\ell}} x\left(T_{\ell}\right)\right) .
$$

Then we find

$$
\begin{aligned}
x(t)= & -\left(T_{\ell}-T_{\ell-1}\right)^{1-u_{\ell}}\left(t-T_{\ell-1}\right)^{u_{\ell}-1} I_{T_{\ell-1}^{+}}^{u_{\ell}} f_{1}\left(T_{\ell}, x\left(T_{\ell}\right), I_{T_{\ell-1}^{+}}^{u_{\ell}} x\left(T_{\ell}\right)\right) \\
& +I_{T_{\ell-1}^{+}}^{u_{\ell}} f_{1}\left(t, x(t), I_{T_{\ell-1}^{+}}^{u_{\ell}} x(t)\right), \quad t \in J_{\ell} .
\end{aligned}
$$


Conversely, let $x \in E_{\ell}$ be a solution of integral equation (7), Regarding the continuity of the unction $t^{\delta} f_{1}$ and Lemma 2.1, we deduce that $x$ is a solution of problem (6).

Our first existence result is based on Theorem 2.1.

Theorem 3.1 Assume that conditions (H1) and (H2) hold and

$$
\frac{2\left(T_{\ell}-T_{\ell-1}\right)^{u_{\ell}-1}\left(T_{\ell}^{1-\delta}-T_{\ell-1}^{1-\delta}\right)}{(1-\delta) \Gamma\left(u_{\ell}\right)}\left(K+L \frac{\left(T_{\ell}-T_{\ell-1}\right)^{u_{\ell}}}{\Gamma\left(u_{\ell}+1\right)}\right)<1 .
$$

Then problem (6) possesses at least one solution on J.

Proof We construct the operator

$$
W: E_{\ell} \rightarrow E_{\ell}
$$

as follows:

$$
\begin{aligned}
W x(t)= & -\left(T_{\ell}-T_{\ell-1}\right)^{1-u_{\ell}}\left(t-T_{\ell-1}\right)^{u_{\ell}-1} I_{T_{\ell-1}^{+}}^{u_{\ell}} f_{1}\left(T_{\ell}, x\left(T_{\ell}\right), I_{T_{\ell-1}^{+}}^{u_{\ell}} x\left(T_{\ell}\right)\right) \\
& +\frac{1}{\Gamma\left(u_{\ell}\right)} \int_{T_{\ell-1}}^{t}(t-s)^{u_{\ell-1}} f_{1}\left(s, x(s), I_{T_{\ell-1}^{+}}^{u_{\ell}} x(s)\right) d s, \quad t \in J_{\ell} .
\end{aligned}
$$

It follows from the properties of fractional integrals and the continuity of function $t^{\delta} f_{1}$ that the operator $W$ is well defined.

Let

$$
R_{\ell} \geq \frac{\frac{2 f^{\star}\left(T_{\ell}-T_{\ell-1}\right)^{u_{\ell}}}{\Gamma\left(u_{\ell}\right)}}{1-\frac{2\left(T_{\ell}-T_{\ell-1}\right)^{u_{\ell}-1}\left(T_{\ell}^{1-\delta}-T_{\ell-1}^{1-\delta}\right)}{(1-\delta) \Gamma\left(u_{\ell}\right)}\left(K+L \frac{\left(T_{\ell}-T_{\ell-1}\right)^{u_{\ell}}}{\Gamma\left(u_{\ell}+1\right)}\right)}
$$

with

$$
f^{\star}=\sup _{t \in J_{\ell}}\left\|f_{1}(t, 0,0)\right\|
$$

We consider the set

$$
B_{R_{\ell}}=\left\{x \in E_{\ell},\|x\|_{E_{\ell}} \leq R_{\ell}\right\} .
$$

Clearly, $B_{R_{\ell}}$ is nonempty, closed, convex, and bounded.

Now we demonstrate that $W$ satisfies the assumptions of Theorem 2.1. We shall prove it in four phases.

Step 1: $W\left(B_{R_{\ell}}\right) \subseteq\left(B_{R_{\ell}}\right)$.

For $x \in B_{R_{\ell}}$, by (H2) we get:

$$
\begin{aligned}
\|W x(t)\| \leq & \frac{\left(T_{\ell}-T_{\ell-1}\right)^{1-u_{\ell}}\left(t-T_{\ell-1}\right)^{u_{\ell}-1}}{\Gamma\left(u_{\ell}\right)} \int_{T_{\ell-1}}^{T_{\ell}}\left(T_{\ell}-s\right)^{u_{\ell}-1}\left\|f_{1}\left(s, x(s), I_{T_{\ell-1}^{+}}^{u_{\ell}} x(s)\right)\right\| d s \\
& +\frac{1}{\Gamma\left(u_{\ell}\right)} \int_{T_{\ell-1}}^{t}(t-s)^{u_{\ell}-1}\left\|f_{1}\left(s, x(s), I_{T_{\ell-1}^{+}}^{u_{\ell}} x(s)\right)\right\| d s
\end{aligned}
$$




$$
\begin{aligned}
\leq & \frac{2}{\Gamma\left(u_{\ell}\right)} \int_{T_{\ell-1}}^{T_{\ell}}\left(T_{\ell}-s\right)^{u_{\ell}-1}\left\|f_{1}\left(s, x(s), I_{T_{\ell-1}^{+}}^{u_{\ell}} x(s)\right)\right\| d s \\
\leq & \frac{2}{\Gamma\left(u_{\ell}\right)} \int_{T_{\ell-1}}^{T_{\ell}}\left(T_{\ell}-s\right)^{u_{\ell}-1}\left\|f_{1}\left(s, x(s), I_{T_{\ell-1}^{+}}^{u_{\ell}} x(s)\right)-f_{1}(s, 0,0)\right\| d s \\
& +\frac{2}{\Gamma\left(u_{\ell}\right)} \int_{T_{\ell-1}}^{T_{\ell}}\left(T_{\ell}-s\right)^{u_{\ell}-1}\left\|f_{1}(s, 0,0)\right\| d s \\
\leq & \frac{2}{\Gamma\left(u_{\ell}\right)} \int_{T_{\ell-1}}^{T_{\ell}}\left(T_{\ell}-s\right)^{u_{\ell}-1} s^{-\delta}\left(K\|x(s)\|+L\left\|I_{T_{\ell-1}^{+}}^{u_{\ell}} x(s)\right\|\right) d s+\frac{2 f^{\star}\left(T_{\ell}-T_{\ell-1}\right)^{u_{\ell}}}{\Gamma\left(u_{\ell}\right)} \\
\leq & \frac{2\left(T_{\ell}-T_{\ell-1}\right)^{u_{\ell}-1}}{\Gamma\left(u_{\ell}\right)} \int_{T_{\ell-1}}^{T_{\ell}} s^{-\delta}\left(K+L \frac{\left(T_{\ell}-T_{\ell-1}\right)^{u_{\ell}}}{\Gamma\left(u_{\ell}+1\right)}\right)\|x(s)\| d s \\
& +\frac{2 f^{\star}\left(T_{\ell}-T_{\ell-1}\right)^{u_{\ell}}}{\Gamma\left(u_{\ell}\right)} \\
\leq & \frac{2\left(T_{\ell}-T_{\ell-1}\right)^{u_{\ell}-1}\left(T_{\ell}^{1-\delta}-T_{\ell-1}^{1-\delta}\right)}{(1-\delta) \Gamma\left(u_{\ell}\right)}\left(K+L \frac{\left(T_{\ell}-T_{\ell-1}\right)^{u_{\ell}}}{\Gamma\left(u_{\ell}+1\right)}\right) R_{\ell} \\
& +\frac{2 f^{\star}\left(T_{\ell}-T_{\ell-1}\right)^{u_{\ell}}}{\Gamma\left(u_{\ell}\right)} \\
\leq & R_{\ell},
\end{aligned}
$$

which means that $W\left(B_{R_{\ell}}\right) \subseteq B_{R_{\ell}}$.

Step 2: $W$ is continuous.

Let a sequence $\left(x_{n}\right)$ converge to $x$ in $E_{\ell}$, and let $t \in J_{\ell}$. Then

$$
\begin{aligned}
\left\|\left(W x_{n}\right)(t)-(W x)(t)\right\| & \\
\leq & \frac{\left(T_{\ell}-T_{\ell-1}\right)^{1-u_{\ell}}\left(t-T_{\ell-1}\right)^{u_{\ell}-1}}{\Gamma\left(u_{\ell}\right)} \int_{T_{\ell-1}}^{T_{\ell}}\left(T_{\ell}-s\right)^{u_{\ell}-1} \| f_{1}\left(s, x_{n}(s), I_{T_{\ell-1}^{+}}^{u_{\ell}} x_{n}(s)\right) \\
& -f_{1}\left(s, x(s), I_{T_{\ell-1}^{+}}^{u_{\ell}} x(s)\right) \| d s \\
& +\frac{1}{\Gamma\left(u_{\ell}\right)} \int_{T_{\ell-1}}^{t}(t-s)^{u_{\ell}-1}\left\|f_{1}\left(s, x_{n}(s), I_{T_{\ell-1}^{+}}^{u_{\ell}} x_{n}(s)\right)-f_{1}\left(s, x(s), I_{T_{\ell-1}^{+}}^{u_{\ell}} x(s)\right)\right\| d s \\
\leq & \frac{\left(T_{\ell}-T_{\ell-1}\right)^{1-u_{\ell}}\left(T_{\ell}-T_{\ell-1}\right)^{u_{\ell}-1}}{\Gamma\left(u_{\ell}\right)} \int_{T_{\ell-1}}^{T_{\ell}}\left(T_{\ell}-s\right)^{u_{\ell}-1} \| f_{1}\left(s, x_{n}(s), I_{T_{\ell-1}^{+}}^{u_{\ell}} x_{n}(s)\right) \\
& -f_{1}\left(s, x(s), I_{T_{\ell-1}^{+}}^{u_{\ell}} x(s)\right) \| d s \\
& +\frac{1}{\Gamma\left(u_{\ell}\right)} \int_{T_{\ell-1}}^{T_{\ell}}(t-s)^{u_{\ell}-1}\left\|f_{1}\left(s, x_{n}(s), I_{T_{\ell-1}^{+}}^{u_{\ell}} x_{n}(s)\right)-f_{1}\left(s, x(s), I_{T_{\ell-1}^{+}}^{u_{\ell}} x(s)\right)\right\| d s \\
\leq & \frac{2}{\Gamma\left(u_{\ell}\right)} \int_{T_{\ell-1}}^{T_{\ell}}\left(T_{\ell}-s\right)^{u_{\ell}-1}\left\|f_{1}\left(s, x_{n}(s), I_{T_{\ell-1}^{+}}^{u_{\ell}} x_{n}(s)\right)-f_{1}\left(s, x(s), I_{T_{\ell-1}^{+}}^{u_{\ell}} x(s)\right)\right\| d s \\
\leq & \left.\frac{2}{\Gamma\left(u_{\ell}\right)} \int_{T_{\ell-1}}^{T_{\ell}} s^{-\delta}\left(T_{\ell}-s\right)^{u_{\ell}-1}\left(K\left\|x_{n}(s)-x(s)\right\|+L I_{T_{\ell-1}^{+}}^{u_{\ell}} \| x_{n}(s)-x(s)\right) \|\right) d s \\
\leq & \frac{2 K}{\Gamma\left(u_{\ell}\right)}\left\|x_{n}-x\right\|_{E_{\ell}} \int_{T_{\ell-1}}^{T_{\ell}} s^{-\delta}\left(T_{\ell}-s\right)^{u_{\ell}-1} d s \\
& +\frac{2 L}{\Gamma\left(u_{\ell}\right)}\left\|I_{T_{\ell-1}^{+}}^{u_{\ell}}\left(x_{n}-x\right)\right\|_{E_{\ell}} \int_{T_{\ell-1}}^{T_{\ell}} s^{-\delta}\left(T_{\ell}-s\right)^{u_{\ell}-1} d s
\end{aligned}
$$




$$
\begin{aligned}
\leq & \frac{2 K}{\Gamma\left(u_{\ell}\right)}\left\|x_{n}-x\right\|_{E_{\ell}} \int_{T_{\ell-1}}^{T_{\ell}} s^{-\delta}\left(T_{\ell}-s\right)^{u_{\ell}-1} d s \\
& +\frac{2 L\left(T_{\ell}-T_{\ell-1}\right)^{u_{\ell}}}{\Gamma\left(u_{\ell}\right) \Gamma\left(u_{\ell}+1\right)}\left\|x_{n}-x\right\|_{E_{\ell}} \int_{T_{\ell-1}}^{T_{\ell}} s^{-\delta}\left(T_{\ell}-s\right)^{u_{\ell}-1} d s \\
\leq & \left(\frac{2 K}{\Gamma\left(u_{\ell}\right)}+\frac{2 L\left(T_{\ell}-T_{\ell-1}\right)^{u_{\ell}}}{\Gamma\left(u_{\ell}\right) \Gamma\left(u_{\ell}+1\right)}\right)\left\|x_{n}-x\right\|_{E_{\ell}} \int_{T_{\ell-1}}^{T_{\ell}} s^{-\delta}\left(T_{\ell}-s\right)^{u_{\ell}-1} d s \\
\leq & \frac{\left(T_{\ell}-T_{\ell-1}\right)^{u_{\ell}-1}\left(T_{\ell}^{1-\delta}-T_{\ell-1}^{1-\delta}\right)}{(1-\delta) \Gamma\left(u_{\ell}\right)}\left(2 K+\frac{2 L\left(T_{\ell}-T_{\ell-1}\right)^{u_{\ell}}}{\Gamma\left(u_{\ell}+1\right)}\right)\left\|x_{n}-x\right\|_{E_{\ell}},
\end{aligned}
$$

that is,

$$
\left\|\left(W x_{n}\right)-(W x)\right\|_{E_{\ell}} \rightarrow 0 \quad \text { as } n \rightarrow \infty
$$

Thus the operator $W$ is continuous on $E_{\ell}$.

Step 3: $W$ is bounded and equicontinuous.

From Step 2 we have $W\left(B_{R_{\ell}}\right)=\left\{W(x): x \in B_{R_{\ell}}\right\} \subset B_{R_{\ell}}$, and hence, for each $x \in B_{R_{\ell}}$, we have $\|W(x)\|_{E_{\ell}} \leq R_{\ell}$, which means that $W\left(B_{R_{\ell}}\right)$ is bounded. It remains to check that $W\left(B_{R_{\ell}}\right)$ is equicontinuous.

For $t_{1}, t_{2} \in J_{\ell}, t_{1}<t_{2}$, and $x \in B_{R_{\ell}}$, we have:

$$
\begin{aligned}
& \left\|(W x)\left(t_{2}\right)-(W x)\left(t_{1}\right)\right\| \\
& =\|-\frac{\left(T_{\ell}-T_{\ell-1}\right)^{1-u_{\ell}}\left(t_{2}-T_{\ell-1}\right)^{u_{\ell}-1}}{\Gamma\left(u_{\ell}\right)} \\
& \times \int_{T_{\ell-1}}^{T_{\ell}}\left(T_{\ell}-s\right)^{u_{\ell}-1} f_{1}\left(s, x(s), I_{T_{\ell-1}^{+}}^{u_{\ell}} x(s)\right) d s \\
& +\frac{1}{\Gamma\left(u_{\ell}\right)} \int_{T_{\ell-1}}^{t_{2}}\left(t_{2}-s\right)^{u_{\ell}-1} f_{1}\left(s, x(s), I_{T_{\ell-1}^{+}}^{u_{\ell}} x(s)\right) d s+\frac{\left(T_{\ell}-T_{\ell-1}\right)^{1-u_{\ell}}\left(t_{1}-T_{\ell-1}\right)^{u_{\ell}-1}}{\Gamma\left(u_{\ell}\right)} \\
& \times \int_{T_{\ell-1}}^{T_{\ell}}\left(T_{\ell}-s\right)^{u_{\ell}-1} f_{1}\left(s, x(s), I_{T_{\ell-1}^{+}}^{u_{\ell}} x(s)\right) d s \\
& -\frac{1}{\Gamma\left(u_{\ell}\right)} \int_{T_{\ell-1}}^{t_{1}}\left(t_{1}-s\right)^{u_{\ell}-1} f_{1}\left(s, x(s), I_{T_{\ell-1}^{+}}^{u_{\ell}} x(s)\right) d s \| \\
& \leq \frac{\left(T_{\ell}-T_{\ell-1}\right)^{1-u_{\ell}}}{\Gamma\left(u_{\ell}\right)}\left(\left(t_{2}-T_{\ell-1}\right)^{u_{\ell}-1}-\left(t_{1}-T_{\ell-1}\right)^{u_{\ell}-1}\right) \\
& \times \int_{T_{\ell-1}}^{T_{\ell}}\left(T_{\ell}-s\right)^{u_{\ell}-1}\left\|f_{1}\left(s, x(s), I_{T_{\ell-1}^{+}}^{u_{\ell}} x(s)\right)\right\| d s \\
& +\frac{1}{\Gamma\left(u_{\ell}\right)} \int_{T_{\ell-1}}^{t_{1}}\left(\left(t_{2}-s\right)^{u_{\ell}-1}-\left(t_{1}-s\right)^{u_{\ell}-1}\right)\left\|f_{1}\left(s, x(s), I_{T_{\ell-1}^{+}}^{u_{\ell}} x(s)\right)\right\| d s \\
& +\frac{1}{\Gamma\left(u_{\ell}\right)} \int_{t_{1}}^{t_{2}}\left(t_{2}-s\right)^{u_{\ell}-1}\left\|f_{1}\left(s, x(s), I_{T_{\ell-1}^{+}}^{u_{\ell}} x(s)\right)\right\| d s \\
& \leq \frac{\left(T_{\ell}-T_{\ell-1}\right)^{1-u_{\ell}}}{\Gamma\left(u_{\ell}\right)}\left(\left(t_{2}-T_{\ell-1}\right)^{u_{\ell}-1}-\left(t_{1}-T_{\ell-1}\right)^{u_{\ell}-1}\right) \\
& \times \int_{T_{\ell-1}}^{T_{\ell}}\left(T_{\ell}-s\right)^{u_{\ell}-1}\left\|f_{1}\left(s, x(s), I_{T_{\ell-1}^{+}}^{u_{\ell}} x(s)\right)-f_{1}(s, 0,0)\right\| d s
\end{aligned}
$$




$$
\begin{aligned}
& +\frac{\left(T_{\ell}-T_{\ell-1}\right)^{1-u_{\ell}}}{\Gamma\left(u_{\ell}\right)}\left(\left(t_{2}-T_{\ell-1}\right)^{u_{\ell}-1}-\left(t_{1}-T_{\ell-1}\right)^{u_{\ell}-1}\right) \int_{T_{\ell-1}}^{T_{\ell}}\left(T_{\ell}-s\right)^{u_{\ell}-1}\left\|f_{1}(s, 0,0)\right\| d s \\
& +\frac{1}{\Gamma\left(u_{\ell}\right)} \int_{T_{\ell-1}}^{t_{1}}\left(\left(t_{2}-s\right)^{u_{\ell}-1}-\left(t_{1}-s\right)^{u_{\ell}-1}\right)\left\|f_{1}\left(s, x(s), I_{T_{\ell-1}^{+}}^{u_{\ell}} x(s)\right)-f_{1}(s, 0,0)\right\| d s \\
& +\frac{1}{\Gamma\left(u_{\ell}\right)} \int_{T_{\ell-1}}^{t_{1}}\left(\left(t_{2}-s\right)^{u_{\ell}-1}-\left(t_{1}-s\right)^{u_{\ell}-1}\right)\left\|f_{1}(s, 0,0)\right\| d s \\
& +\frac{1}{\Gamma\left(u_{\ell}\right)} \int_{t_{1}}^{t_{2}}\left(t_{2}-s\right)^{u_{\ell}-1}\left\|f_{1}\left(s, x(s), I_{T_{\ell-1}^{+}}^{u_{\ell}} x(s)\right)-f_{1}(s, 0,0)\right\| d s \\
& +\frac{1}{\Gamma\left(u_{\ell}\right)} \int_{t_{1}}^{t_{2}}\left(t_{2}-s\right)^{u_{\ell}-1}\left\|f_{1}(s, 0,0)\right\| d s \\
& \leq \frac{\left(T_{\ell}-T_{\ell-1}\right)^{1-u_{\ell}}}{\Gamma\left(u_{\ell}\right)}\left(\left(t_{2}-T_{\ell-1}\right)^{u_{\ell}-1}-\left(t_{1}-T_{\ell-1}\right)^{u_{\ell}-1}\right) \\
& \times \int_{T_{\ell-1}}^{T_{\ell}}\left(T_{\ell}-s\right)^{u_{\ell}-1} s^{-\delta}\left(K\|x(s)\|+L\left\|I_{T_{\ell-1}^{+}}^{u_{\ell}} x(s)\right\|\right) d s \\
& +\frac{f^{\star}\left(T_{\ell}-T_{\ell-1}\right)^{1-u_{\ell}}}{\Gamma\left(u_{\ell}\right)}\left(\left(t_{2}-T_{\ell-1}\right)^{u_{\ell}-1}-\left(t_{1}-T_{\ell-1}\right)^{u_{\ell}-1}\right) \int_{T_{\ell-1}}^{T_{\ell}}\left(T_{\ell}-s\right)^{u_{\ell}-1} d s \\
& +\frac{1}{\Gamma\left(u_{\ell}\right)} \int_{T_{\ell-1}}^{t_{1}} s^{-\delta}\left(\left(t_{2}-s\right)^{u_{\ell}-1}-\left(t_{1}-s\right)^{u_{\ell}-1}\right)\left(K\|x(s)\|+L\left\|I_{T_{\ell-1}^{+}}^{u_{\ell}} x(s)\right\|\right) d s \\
& +\frac{f^{\star}}{\Gamma\left(u_{\ell}\right)} \int_{T_{\ell-1}}^{t_{1}}\left(\left(t_{2}-s\right)^{u_{\ell}-1}-\left(t_{1}-s\right)^{u_{\ell}-1}\right) d s \\
& +\frac{1}{\Gamma\left(u_{\ell}\right)} \int_{t_{1}}^{t_{2}} s^{-\delta}\left(t_{2}-s\right)^{u_{\ell}-1}\left(K\|x(s)\|+L\left\|I_{T_{\ell-1}^{+}}^{u_{\ell}} x(s)\right\|\right) d s \\
& +\frac{f^{\star}}{\Gamma\left(u_{\ell}\right)} \int_{t_{1}}^{t_{2}}\left(t_{2}-s\right)^{u_{\ell}-1} d s \\
& \leq \frac{1}{\Gamma\left(u_{\ell}\right)}\left(\left(t_{2}-T_{\ell-1}\right)^{u_{\ell}-1}-\left(t_{1}-T_{\ell-1}\right)^{u_{\ell}-1}\right)\left(K\|x\|_{E_{\ell}}+L\left\|I_{T_{\ell-1}^{+}}^{u_{\ell}} x\right\|_{E_{\ell}}\right) \int_{T_{\ell-1}}^{T_{\ell}} s^{-\delta} d s \\
& +\frac{f^{\star}\left(T_{\ell}-T_{\ell-1}\right)}{\Gamma\left(u_{\ell}+1\right)}\left(\left(t_{2}-T_{\ell-1}\right)^{u_{\ell}-1}-\left(t_{1}-T_{\ell-1}\right)^{u_{\ell}-1}\right) \\
& +\frac{1}{\Gamma\left(u_{\ell}\right)}\left(K\|x\|_{E_{\ell}}+L\left\|I_{T_{\ell-1}^{+}}^{u_{\ell}} x\right\|_{E_{\ell}}\right) \int_{T_{\ell-1}}^{t_{1}} s^{-\delta}\left(\left(t_{2}-t_{1}\right)^{u_{\ell}-1}\right) d s \\
& +\frac{f^{\star}}{\Gamma\left(u_{\ell}\right)}\left(\frac{\left(t_{2}-T_{\ell-1}\right)^{u_{\ell}}}{u_{\ell}}-\frac{\left(t_{2}-t_{1}\right)^{u_{\ell}}}{u_{\ell}}-\frac{\left(t_{1}-T_{\ell-1}\right)^{u_{\ell}}}{u_{\ell}}\right) \\
& +\frac{\left(t_{2}-t_{1}\right)^{u_{\ell}-1}}{\Gamma\left(u_{\ell}\right)}\left(K\|x\|_{E_{\ell}}+L\left\|I_{T_{\ell-1}^{+}}^{u_{\ell}} x\right\|_{E_{\ell}}\right) \int_{t_{1}}^{t_{2}} s^{-\delta} d s+\frac{f^{\star}}{\Gamma\left(u_{\ell}\right)} \frac{\left(t_{2}-t_{1}\right)^{u_{\ell}}}{u_{\ell}} \\
& \leq \frac{T_{\ell}^{1-\delta}-T_{\ell-1}^{1-\delta}}{(1-\delta) \Gamma\left(u_{\ell}\right)}\left(\left(t_{2}-T_{\ell-1}\right)^{u_{\ell}-1}-\left(t_{1}-T_{\ell-1}\right)^{u_{\ell}-1}\right) \\
& \times\left(K\|x\|_{E_{\ell}}+L \frac{\left(T_{\ell}-T_{\ell-1}\right)^{u_{\ell}}}{\Gamma\left(u_{\ell}+1\right)}\|x\|_{E_{\ell}}\right) \\
& +\frac{f^{\star}\left(T_{\ell}-T_{\ell-1}\right)}{\Gamma\left(u_{\ell}+1\right)}\left(\left(t_{2}-T_{\ell-1}\right)^{u_{\ell}-1}-\left(t_{1}-T_{\ell-1}\right)^{u_{\ell}-1}\right) \\
& +\left(\frac{\left(t_{1}^{1-\delta}-T_{\ell-1}^{1-\delta}\right)\left(t_{2}-t_{1}\right)^{u_{\ell}-1}}{(1-\delta) \Gamma\left(u_{\ell}\right)}\right)\left(K\|x\|_{E_{\ell}}+L \frac{\left(T_{\ell}-T_{\ell-1}\right)^{u_{\ell}}}{\Gamma\left(u_{\ell}+1\right)}\|x\|_{E_{\ell}}\right)
\end{aligned}
$$




$$
\begin{aligned}
& +\frac{f^{\star}}{\Gamma\left(u_{\ell}+1\right)}\left(\left(t_{2}-T_{\ell-1}\right)^{u_{\ell}}-\left(t_{2}-t_{1}\right)^{u_{\ell}}-\left(t_{1}-T_{\ell-1}\right)^{u_{\ell}}\right) \\
& +\frac{\left(t_{2}{ }^{1-\delta}-t_{1}{ }^{1-\delta}\right)\left(t_{2}-t_{1}\right)^{u_{\ell}-1}}{(1-\delta) \Gamma\left(u_{\ell}\right)}\left(K\|x\|_{E_{\ell}}+L \frac{\left(T_{\ell}-T_{\ell-1}\right)^{u_{\ell}}}{\Gamma\left(u_{\ell}+1\right)}\|x\|_{E_{\ell}}\right)+\frac{f^{\star}\left(t_{2}-t_{1}\right)^{u_{\ell}}}{\Gamma\left(u_{\ell}+1\right)} \\
& \leq\left(\frac{T_{\ell}^{1-\delta}-T_{\ell-1}{ }^{1-\delta}}{(1-\delta) \Gamma\left(u_{\ell}\right)}\left(K+L \frac{\left(T_{\ell}-T_{\ell-1}\right)^{u_{\ell}}}{\Gamma\left(u_{\ell}+1\right)}\right)\|x\|_{E_{\ell}}+\frac{f^{\star}\left(T_{\ell}-T_{\ell-1}\right)}{\Gamma\left(u_{\ell}+1\right)}\right) \\
& \quad \times\left(\left(t_{2}-T_{\ell-1}\right)^{u_{\ell}-1}-\left(t_{1}-T_{\ell-1}\right)^{u_{\ell}-1}\right) \\
& +\left(\frac{t_{2}^{1-\delta}-T_{\ell-1}{ }^{1-\delta}}{(1-\delta) \Gamma\left(u_{\ell}\right)}\left(K+L \frac{\left(T_{\ell}-T_{\ell-1}\right)^{u_{\ell}}}{\Gamma\left(u_{\ell}+1\right)}\right)\|x\|_{E_{\ell}}\right)\left(t_{2}-t_{1}\right)^{u_{\ell}-1} \\
& +\frac{f^{\star}}{\Gamma\left(u_{\ell}+1\right)}\left(\left(t_{2}-T_{\ell-1}\right)^{u_{\ell}}-\left(t_{1}-T_{\ell-1}\right)^{u_{\ell}}\right) .
\end{aligned}
$$

Hence $\left\|(W x)\left(t_{2}\right)-(W x)\left(t_{1}\right)\right\|_{E_{\ell}} \rightarrow 0$ as $\left|t_{2}-t_{1}\right| \rightarrow 0$, which implies that $T\left(B_{R_{\ell}}\right)$ is equicontinuous.

Step 4: $W$ is a $k$-set contraction.

For $U \in B_{R_{\ell}}$ and $t \in J_{\ell}$, we have:

$$
\begin{aligned}
\zeta(W(U)(t))= & \zeta((W x)(t), x \in U) \\
\leq & \left\{\frac{\left(T_{\ell}-T_{\ell-1}\right)^{1-u_{\ell}}\left(t-T_{\ell-1}\right)^{u_{\ell}-1}}{\Gamma\left(u_{\ell}\right)} \int_{T_{\ell-1}}^{T_{\ell}}\left(T_{\ell}-s\right)^{u_{\ell}-1} \zeta f_{1}\left(s, x(s), I_{T_{\ell-1}^{+}}^{u_{\ell}} x(s)\right) d s\right. \\
& \left.+\frac{1}{\Gamma\left(u_{\ell}\right)} \int_{T_{\ell-1}}^{t}(t-s)^{u_{\ell}-1} \zeta f_{1}\left(s, x(s), I_{T_{\ell-1}^{+}}^{u_{\ell}} x(s)\right) d s, x \in U\right\} .
\end{aligned}
$$

Then Remark 3.1 implies that, for each $s \in J_{i}$,

$$
\begin{aligned}
\zeta( & W(U)(t)) \\
\leq & \left\{\frac{\left(T_{\ell}-T_{\ell-1}\right)^{1-u_{\ell}}\left(t-T_{\ell-1}\right)^{u_{\ell}-1}}{\Gamma\left(u_{\ell}\right)}\right. \\
& \times \int_{T_{\ell-1}}^{T_{\ell}}\left(T_{\ell}-s\right)^{u_{\ell}-1}\left[K \widehat{\zeta}(U) \int_{T_{\ell-1}}^{T_{\ell}} s^{-\delta} d s+L \frac{\left(T_{\ell}-T_{\ell-1}\right)^{u_{\ell}}}{\Gamma\left(u_{\ell}+1\right)} \widehat{\zeta}(U) \int_{T_{\ell-1}}^{T_{\ell}} s^{-\delta} d s\right] \\
& +\frac{1}{\Gamma\left(u_{\ell}\right)} \int_{T_{\ell-1}}^{t}(t-s)^{u_{\ell}-1}\left[K \widehat{\zeta}(U) \int_{T_{\ell-1}}^{t} s^{-\delta} d s\right. \\
& \left.\left.+L \frac{\left(T_{\ell}-T_{\ell-1}\right)^{u_{\ell}}}{\Gamma\left(u_{\ell}+1\right)} \widehat{\zeta}(U) \int_{T_{\ell-1}}^{t} s^{-\delta} d s\right], x \in U\right\} \\
\leq & \left\{\frac{\left(t-T_{\ell-1}\right)^{u_{\ell}-1}}{\Gamma\left(u_{\ell}\right)} \int_{T_{\ell-1}}^{T_{\ell}}\left[K \widehat{\zeta}(U) \int_{T_{\ell-1}}^{T_{\ell}} s^{-\delta} d s+L \frac{\left(T_{\ell}-T_{\ell-1}\right)^{u_{\ell}}}{\Gamma\left(u_{\ell}+1\right)} \widehat{\zeta}(U) \int_{T_{\ell-1}}^{T_{\ell}} s^{-\delta} d s\right]\right. \\
& +\frac{\left(t-T_{\ell-1}\right)^{u_{\ell}-1}}{\Gamma\left(u_{\ell}\right)} \int_{T_{\ell-1}}^{t}\left[K \widehat{\zeta}(U) \int_{T_{\ell-1}}^{t} s^{-\delta} d s\right. \\
& \left.\left.+L \frac{\left(T_{\ell}-T_{\ell-1}\right)^{u_{\ell}}}{\Gamma\left(u_{\ell}+1\right)} \widehat{\zeta}(U) \int_{T_{\ell-1}}^{t} s^{-\delta} d s\right], x \in U\right\} \\
\leq & \frac{\left[\left(T_{\ell}^{1-\delta}-T_{\ell-1}^{1-\delta}\right)+\left(t^{1-\delta}-T_{\ell-1}^{1-\delta}\right)\right]\left(t-T_{\ell-1}\right)^{u_{\ell}-1}}{(1-\delta) \Gamma\left(u_{\ell}\right)}\left(K+L \frac{\left(T_{\ell}-T_{\ell-1}\right)^{u_{\ell}}}{\Gamma\left(u_{\ell}+1\right)}\right) \widehat{\zeta}(U)
\end{aligned}
$$




$$
\leq \frac{2\left(T_{\ell}^{1-\delta}-T_{\ell-1}^{1-\delta}\right)\left(T_{\ell}-T_{\ell-1}\right)^{u_{\ell}-1}}{(1-\delta) \Gamma\left(u_{\ell}\right)}\left(K+L \frac{\left(T_{\ell}-T_{\ell-1}\right)^{u_{\ell}}}{\Gamma\left(u_{\ell}+1\right)}\right) \widehat{\zeta}(U)
$$

Therefore we have:

$$
\widehat{\zeta}(W U) \leq \frac{2\left(T_{\ell}^{1-\delta}-T_{\ell-1}^{1-\delta}\right)\left(T_{\ell}-T_{\ell-1}\right)^{u_{\ell}-1}}{(1-\delta) \Gamma\left(u_{\ell}\right)}\left(K+L \frac{\left(T_{\ell}-T_{\ell-1}\right)^{u_{\ell}}}{\Gamma\left(u_{\ell}+1\right)}\right) \widehat{\zeta}(U) .
$$

Consequently, from (8) we deduce that $W$ forms a set contraction. Hence by Theorem 2.1 problem (6) has at least a solution $\tilde{x_{\ell}}$ in $B_{R_{\ell}}$.

Let

$$
x_{\ell}= \begin{cases}0, & t \in\left[0, T_{\ell-1}\right], \\ \tilde{x}_{\ell}, & t \in J_{\ell} .\end{cases}
$$

We know that $x_{\ell} \in C\left(\left[0, T_{\ell}\right], X\right)$ defined by (10) satisfies the equation

$$
\frac{d^{2}}{d t^{2}}\left(\int_{0}^{T_{1}} \frac{(t-s)^{1-u_{1}}}{\Gamma\left(2-u_{1}\right)} x_{\ell}(s) d s+\cdots+\int_{T_{\ell-1}}^{t} \frac{(t-s)^{1-u_{\ell}}}{\Gamma\left(2-u_{\ell}\right)} x_{\ell}(s) d s\right)=f_{1}\left(s, x_{\ell}(s), I_{0^{+}}^{u_{\ell}} x_{\ell}(s)\right)
$$

for $t \in J_{\ell}$, which means that $x_{\ell}$ is a solution of (5) with $x_{\ell}(0)=0$ and $x_{\ell}\left(T_{\ell}\right)=\widetilde{x}_{\ell}\left(T_{\ell}\right)=0$. Then

$$
x(t)= \begin{cases}x_{1}(t), & t \in J_{1}, \\ x_{2}(t)= \begin{cases}0, & t \in J_{1}, \\ \tilde{x}_{2}, & t \in J_{2},\end{cases} \\ \vdots \\ x_{n}(t)= \begin{cases}0, & t \in\left[0, T_{\ell-1}\right], \\ \tilde{x}_{\ell}, & t \in J_{\ell},\end{cases} \end{cases}
$$

forms a solution of BVP (1).

\section{Ulam-Hyers-Rassias stability}

Theorem 4.1 Assume (H1), (H2), (8), and

(H3) $\vartheta \in C\left(J_{\ell}, X\right)$ is an increasing function, and there exists $\lambda_{\vartheta}>0$ such that

$$
I_{T_{\ell-1}{ }^{+}}^{u_{\ell}} \vartheta(t) \leq \lambda_{\vartheta}(t) \vartheta(t) \quad \text { for all } t \in J_{\ell} .
$$

Then equation of (1) is UHR stable with respect to $\vartheta$.

Proof Let $z \in C\left(J_{\ell}, X\right)$ be a solution of the inequality

$$
\left\|D_{T_{\ell-1}{ }^{+}}^{u_{\ell}} z(t)-f_{1}\left(t, z(t), I_{T_{\ell-1}+}^{u_{\ell}} z(t)\right)\right\| \leq \epsilon \vartheta(t), \quad t \in J_{\ell} .
$$

Let $x \in C\left(J_{\ell}, X\right)$ be a solution of the problem

$$
D_{T_{\ell-1}+}^{u_{\ell}} x(t)=f_{1}\left(t, x(t), I_{T_{\ell-1}+}^{u_{\ell}} x(t)\right), \quad t \in J_{\ell} .
$$




$$
x\left(T_{\ell-1}\right)=0, \quad x\left(T_{\ell}\right)=0
$$

By Lemma 3.1 we have:

$$
\begin{aligned}
x(t)= & -\frac{\left(T_{\ell}-T_{\ell-1}\right)^{1-u_{\ell}}\left(t-T_{\ell-1}\right)^{u_{\ell}-1}}{\Gamma\left(u_{\ell}\right)} \int_{T_{\ell-1}}^{T_{\ell}}\left(T_{\ell}-s\right)^{u_{\ell-1}} f_{1}\left(s, x(s), I_{T_{\ell-1}^{+}}^{u_{\ell}} x(s)\right) d s \\
& +\frac{1}{\Gamma\left(u_{\ell}\right)} \int_{T_{\ell-1}}^{t}(t-s)^{u_{\ell-1}} f_{1}\left(s, x(s), I_{T_{\ell-1}^{+}}^{u_{\ell}} x(s)\right) d s .
\end{aligned}
$$

By integration of (11) from (H3) we obtain:

$$
\begin{aligned}
& \| z(t)+\frac{\left(T_{\ell}-T_{\ell-1}\right)^{1-u_{\ell}}\left(t-T_{\ell-1}\right)^{u_{\ell}-1}}{\Gamma\left(u_{\ell}\right)} \int_{T_{\ell-1}}^{T_{\ell}}\left(T_{\ell}-s\right)^{u_{\ell-1}} f_{1}\left(s, z(s), I_{T_{\ell-1}^{+}}^{u_{\ell}} z(s)\right) d s \\
&-\frac{1}{\Gamma\left(u_{\ell}\right)} \int_{T_{\ell-1}}^{t}(t-s)^{u_{\ell-1}} f_{1}\left(s, z(s), I_{T_{\ell-1}^{+}}^{u_{\ell}} z(s)\right) d s \| \\
& \leq \epsilon \int_{T_{\ell-1}}^{t} \frac{(t-s)^{u(i)-1}}{\Gamma(u(i))} \vartheta(s) d s \\
& \leq \epsilon \lambda_{\vartheta(t)} \vartheta(t) .
\end{aligned}
$$

On the other hand, for each $t \in J_{\ell}$, we have:

$$
\begin{aligned}
& \|z(t)-x(t)\| \\
& =\| z(t)+\frac{\left(T_{\ell}-T_{\ell-1}\right)^{1-u_{\ell}}\left(t-T_{\ell-1}\right)^{u_{\ell}-1}}{\Gamma\left(u_{\ell}\right)} \int_{T_{\ell-1}}^{T_{\ell}}\left(T_{\ell}-s\right)^{u_{\ell-1}} f_{1}\left(s, x(s), I_{T_{\ell-1}^{+}}^{u_{\ell}} x(s)\right) d s \\
& -\frac{1}{\Gamma\left(u_{\ell}\right)} \int_{T_{\ell-1}}^{t}(t-s)^{u_{\ell-1}} f_{1}\left(s, x(s), I_{T_{\ell-1}^{+}}^{u_{\ell}} x(s)\right) d s \| \\
& =\| z(t)+\frac{\left(T_{\ell}-T_{\ell-1}\right)^{1-u_{\ell}}\left(t-T_{\ell-1}\right)^{u_{\ell}-1}}{\Gamma\left(u_{\ell}\right)} \int_{T_{\ell-1}}^{T_{\ell}}\left(T_{\ell}-s\right)^{u_{\ell-1}} f_{1}\left(s, z(s), I_{T_{\ell-1}^{+}}^{u_{\ell}} z(s)\right) d s \\
& -\frac{1}{\Gamma\left(u_{\ell}\right)} \int_{T_{\ell-1}}^{t}(t-s)^{u_{\ell-1}} f_{1}\left(s, z(s), I_{T_{\ell-1}^{+}}^{u_{\ell}} z(s)\right) d s \| \\
& +\frac{\left(T_{\ell}-T_{\ell-1}\right)^{1-u_{\ell}}\left(t-T_{\ell-1}\right)^{u_{\ell}-1}}{\Gamma\left(u_{\ell}\right)} \int_{T_{\ell-1}}^{T_{\ell}}\left(T_{\ell}-s\right)^{u_{\ell}-1} \\
& \left\|f_{1}\left(s, z(s), I_{T_{\ell-1}^{+}}^{u_{\ell}} z\right)-f_{1}\left(s, x(s), I_{T_{\ell-1}^{+}}^{u_{\ell}} x\right)\right\| d s \\
& +\frac{1}{\Gamma\left(u_{\ell}\right)} \int_{T_{\ell-1}}^{t}(t-s)^{u_{\ell}-1}\left\|f_{1}\left(s, z(s), I_{T_{\ell-1}^{+}}^{u_{\ell}} z\right)-f_{1}\left(s, x(s), I_{T_{\ell-1}^{+}}^{u_{\ell}} x\right)\right\| d s \\
& \leq \lambda_{\vartheta(t)} \epsilon \vartheta(t)+\frac{\left(T_{\ell}-T_{\ell-1}\right)^{1-u_{\ell}}\left(t-T_{\ell-1}\right)^{u_{\ell}-1}}{\Gamma\left(u_{\ell}\right)} \\
& \times \int_{T_{\ell-1}}^{T_{\ell}}\left(T_{\ell}-s\right)^{u_{\ell}-1} s^{-\delta}\left(K\|z(s)-x(s)\|+L I_{T_{\ell-1}^{+}}^{u_{\ell}}\|z(s)-x(s)\|\right) d s \\
& +\frac{1}{\Gamma\left(u_{\ell}\right)} \int_{T_{\ell-1}}^{t}(t-s)^{u_{\ell}-1} s^{-\delta}\left(K\|z(s)-x(s)\|+L I_{T_{\ell-1}^{+}}^{u_{\ell}}\|z(s)-x(s)\|\right) d s \\
& \leq \lambda_{\vartheta(t)} \epsilon \vartheta(t)+\frac{\left(T_{\ell}-T_{\ell-1}\right)^{u_{\ell}-1}}{\Gamma\left(u_{\ell}\right)}\left(K\|z-x\|_{E_{\ell}}+L I_{T_{\ell-1}^{+}}^{u_{\ell}}\|z-x\|_{E_{\ell}}\right) \int_{T_{\ell-1}}^{T_{\ell}} s^{-\delta} d s
\end{aligned}
$$




$$
\begin{aligned}
& +\frac{\left(T_{\ell}-T_{\ell-1}\right)^{u_{\ell}-1}}{\Gamma\left(u_{\ell}\right)}\left(K\|z-x\|_{E_{\ell}}+L I_{T_{\ell-1}^{+}}^{u_{\ell}}\|z-x\|_{E_{\ell}}\right) \int_{T_{\ell-1}}^{t} s^{-\delta} d s \\
\leq & \lambda_{\vartheta(t)} \epsilon \vartheta(t)+\frac{\left(T_{\ell}-T_{\ell-1}\right)^{u_{\ell}-1}\left(T_{\ell}^{1-\delta}-T_{\ell-1}^{1-\delta}\right)}{(1-\delta) \Gamma\left(u_{\ell}\right)} \\
& \times\left(K\|z-x\|_{E_{\ell}}+L \frac{\left(T_{\ell}-T_{\ell-1}\right)^{u_{\ell}}}{\Gamma\left(u_{\ell}+1\right)}\|z-x\|_{E_{\ell}}\right) \\
& +\frac{\left(T_{\ell}-T_{\ell-1}\right)^{u_{\ell}-1}\left(t^{1-\delta}-T_{\ell-1}^{1-\delta}\right)}{(1-\delta) \Gamma\left(u_{\ell}\right)}\left(K\|z-x\|_{E_{\ell}}+L \frac{\left(T_{\ell}-T_{\ell-1}\right)^{u_{\ell}}}{\Gamma\left(u_{\ell}+1\right)}\|z-x\|_{E_{\ell}}\right) \\
\leq & \lambda_{\vartheta(t)} \epsilon \vartheta(t)+\frac{2\left(T_{\ell}-T_{\ell-1}\right)^{u_{\ell}-1}\left(T_{\ell}^{1-\delta}-T_{\ell-1}^{1-\delta}\right)}{(1-\delta) \Gamma\left(u_{\ell}\right)}\left(K+L \frac{\left(T_{\ell}-T_{\ell-1}\right)^{u_{\ell}}}{\Gamma\left(u_{\ell}+1\right)}\right)\|z-x\|_{E_{\ell}} .
\end{aligned}
$$

Then

$$
\|z-y\|_{E_{\ell}}\left(1-\frac{2\left(T_{\ell}^{1-\delta}-T_{\ell-1}^{1-\delta}\right)\left(T_{\ell}-T_{\ell-1}\right)^{u_{\ell}-1}}{(1-\delta) \Gamma\left(u_{\ell}\right)}\left(K+L \frac{\left(T_{\ell}-T_{\ell-1}\right)^{u_{\ell}}}{\Gamma\left(u_{\ell}+1\right)}\right)\right) \leq \lambda_{\vartheta(t)} \in \vartheta(t)
$$

For each $t \in J_{\ell}$, we obtain:

$$
\begin{aligned}
\|z-y\|_{E_{\ell}} \leq & \frac{\lambda_{\vartheta(t)} \in \vartheta(t)}{\left(1-\frac{2\left(T_{\ell}{ }^{1-\delta}-T_{\ell-1}{ }^{1-\delta}\right)\left(T_{\ell}-T_{\ell-1}\right)^{u_{\ell}-1}}{(1-\delta) \Gamma\left(u_{\ell}\right)}\left(K+L \frac{\left(T_{\ell}-T_{\ell-1}\right)_{\ell}}{\Gamma\left(u_{\ell}+1\right)}\right)\right)} \\
= & {\left[1-\frac{2\left(T_{\ell}^{1-\delta}-T_{\ell-1}{ }^{1-\delta}\right)\left(T_{\ell}-T_{\ell-1}\right)^{u_{\ell}-1}}{(1-\delta) \Gamma\left(u_{\ell}\right)}\left(K+L \frac{\left(T_{\ell}-T_{\ell-1}\right)^{u_{\ell}}}{\Gamma\left(u_{\ell}+1\right)}\right)\right]^{-1} } \\
& \times \lambda_{\vartheta(t)} \in \vartheta(t):=c_{f_{1}} \in \vartheta(t) .
\end{aligned}
$$

Then the equation in (6) is UHR stable with respect to $\vartheta$ for each $\ell \in\{1,2, \ldots, n\}$.

Consequently, the equation in (1) is UHR stable with respect to $\vartheta$.

\section{Example}

In this example, we deal with the fractional boundary value problem

$$
\left\{\begin{array}{l}
D_{0^{+}}^{u(t)} x(t)=\frac{t^{-\frac{1}{3}} e^{-t}}{\left(e^{\frac{t^{2}}{1+t}}+4 e^{2 t}+1\right)\left(1+|x(t)|+\left|I_{0}^{u(t)} x(t)\right|\right)}, \quad t \in J:=[0,2] \\
x(0)=0, \quad x(2)=0
\end{array}\right.
$$

Let

$$
\begin{aligned}
& f_{1}(t, y, z)=\frac{t^{-\frac{1}{3}} e^{-t}}{\left(e^{e^{2}+t}+4 e^{2 t}+1\right)(1+y+z)}, \quad(t, y, z) \in[0,2] \times[0,+\infty) \times[0,+\infty), \\
& u(t)= \begin{cases}\frac{3}{2}, & t \in J_{1}:=[0,1], \\
\frac{9}{5}, & \left.\left.t \in J_{2}:=\right] 1,2\right] .\end{cases}
\end{aligned}
$$

Then we have:

$$
t^{\frac{1}{3}}\left|f_{1}\left(t, y_{1}, z_{1}\right)-f_{1}\left(t, y_{2}, z_{2}\right)\right|=\left|\frac{e^{-t}}{\left(e^{e^{\frac{t^{2}}{1+t}}}+4 e^{2 t}+1\right)}\left(\frac{1}{1+y_{1}+z_{1}}-\frac{1}{1+y_{2}+z_{2}}\right)\right|
$$




$$
\begin{aligned}
& \leq \frac{e^{-t}\left(\left|y_{1}-y_{2}\right|+\left|z_{1}-z_{2}\right|\right)}{\left(e^{\frac{t^{2}}{1+t}}+4 e^{2 t}+1\right)\left(1+y_{1}+z_{1}\right)\left(1+y_{2}+z_{2}\right)} \\
& \leq \frac{e^{-t}}{\left(e^{\frac{t^{2}}{1+t}}+4 e^{2 t}+1\right)}\left(\left|y_{1}-y_{2}\right|+\left|z_{1}-z_{2}\right|\right) \\
& \leq \frac{1}{(e+5)}\left|y_{1}-y_{2}\right|+\frac{1}{(e+5)}\left|z_{1}-z_{2}\right| .
\end{aligned}
$$

Thus (H2) holds with $\delta=\frac{1}{3}$ and $K=L=\frac{1}{e+5}$.

By (13) the equation of problem (12) can be divided into two expressions as follows:

$$
\begin{array}{ll}
D_{0^{+}}^{\frac{3}{2}} x(t)=\frac{t^{-\frac{1}{3}} e^{-t}}{\left(e^{\frac{t^{2}}{1+t}}+4 e^{2 t}+1\right)\left(1+|x(t)|+\left|I_{0}^{\frac{3}{2}} x(t)\right|\right)}, & t \in J_{1}, \\
D_{1^{+}}^{\frac{9}{5}} x(t)=\frac{t^{-\frac{1}{3}} e^{-t}}{\left(e^{e^{\frac{t^{2}}{+1}}}+4 e^{2 t}+1\right)\left(1+|x(t)|+\left|I_{0}^{\frac{9}{5}} x(t)\right|\right)}, & t \in J_{2} .
\end{array}
$$

For $t \in J_{1}$, problem (12) is equivalent to the problem

$$
\left\{\begin{array}{l}
D_{0^{+}}^{\frac{3}{2}} x(t)=\frac{t^{-\frac{1}{3}} e^{-t}}{\left(e^{\frac{t^{2}}{1+t}}+4 e^{2 t}+1\right)\left(1+|x(t)|+\left|I_{0}^{\frac{3}{2}} x(t)\right|\right)}, \quad t \in J_{1}, \\
x(0)=0, \quad x(1)=0 .
\end{array}\right.
$$

Next, we prove that condition (8) is fulfilled.

$$
\begin{gathered}
\frac{2\left(T_{1}{ }^{1-\delta}-T_{0}{ }^{1-\delta}\right)\left(T_{1}-T_{0}\right)^{u_{1}-1}}{(1-\delta) \Gamma\left(u_{1}\right)}\left(K+\frac{L\left(T_{1}-T_{0}\right)^{u_{1}}}{\Gamma\left(u_{1}+1\right)}\right) \\
=\frac{2}{\frac{2}{3}(e+5) \Gamma\left(\frac{3}{2}\right)}\left(1+\frac{1}{\Gamma\left(\frac{5}{2}\right)}\right) \simeq 0.7685<1 .
\end{gathered}
$$

Let $\vartheta(t)=t^{\frac{1}{2}}$. Then

$$
\begin{aligned}
I_{0^{+}}^{u_{1}} \vartheta(t) & =\frac{1}{\Gamma\left(\frac{3}{2}\right)} \int_{0}^{t}(t-s)^{\frac{1}{2}} s^{\frac{1}{2}} d s \\
& \leq \frac{1}{\Gamma\left(\frac{3}{2}\right)} \int_{0}^{t}(t-s)^{\frac{1}{2}} d s \\
& \leq \frac{2}{3 \Gamma\left(\frac{3}{2}\right)} \vartheta(t):=\lambda_{\vartheta(t)} \vartheta(t) .
\end{aligned}
$$

Thus (H3) is satisfied with $\vartheta(t)=t^{\frac{1}{2}}$ and $\lambda_{\vartheta(t)}=\frac{2}{3 \Gamma\left(\frac{3}{2}\right)}$.

By Theorem 3.1 problem (14) has a solution $x_{1} \in E_{1}$, and by Theorem 4.1 the equation in (14) is UHR stable.

For $t \in J_{2}$, problem (12) can be written as follows:

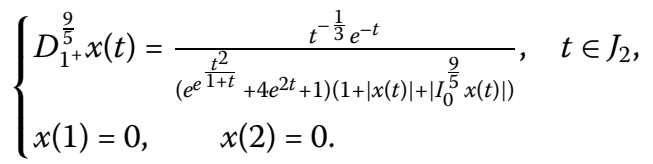


We see that

$$
\begin{gathered}
\frac{2\left(T_{2}{ }^{1-\delta}-T_{1}{ }^{1-\delta}\right)\left(T_{2}-T_{1}\right)^{u_{2}-1}}{(1-\delta) \Gamma\left(u_{2}\right)}\left(K+\frac{L\left(T_{2}-T_{1}\right)^{u_{2}}}{\Gamma\left(u_{2}+1\right)}\right) \\
=\frac{2\left(2^{\frac{2}{3}}-1\right)}{\frac{2}{3} \Gamma\left(\frac{9}{5}\right)} \frac{1}{e+5}\left(1+\frac{1}{\Gamma\left(\frac{14}{5}\right)}\right) \simeq 0.3913<1 .
\end{gathered}
$$

As a result, condition (8) is satisfied. Moreover,

$$
\begin{aligned}
I_{1^{+}}^{u_{2}} \vartheta(t) & =\frac{1}{\Gamma\left(\frac{9}{5}\right)} \int_{1}^{t}(t-s)^{\frac{4}{5}} s^{\frac{1}{2}} d s \\
& \leq \frac{1}{\Gamma\left(\frac{9}{5}\right)} \int_{1}^{t}(t-s)^{\frac{4}{5}} d s \\
& \leq \frac{5}{9 \Gamma\left(\frac{9}{5}\right)} \vartheta(t):=\lambda_{\vartheta(t)} \vartheta(t) .
\end{aligned}
$$

Thus (H3) is fulfilled with $\vartheta(t)=t^{\frac{1}{2}}$ and $\lambda_{\vartheta(t)}=\frac{5}{9 \Gamma\left(\frac{9}{5}\right)}$.

By Theorem 3.1 problem (15) possesses a solution $\widetilde{x}_{2} \in E_{2}$, Further, Theorem 4.1 yields that (15) is UHR stable.

It is known that

$$
x_{2}(t)= \begin{cases}0, & t \in J_{1} \\ \widetilde{x}_{2}(t), & t \in J_{2} .\end{cases}
$$

As a result, by Definition 3.1 the boundary value problem (12) has a solution

$$
x(t)= \begin{cases}x_{1}(t), & t \in J_{1}, \\ x_{2}(t)= \begin{cases}0, & t \in J_{1}, \\ \widetilde{x}_{2}(t), & t \in J_{2} .\end{cases} \end{cases}
$$

In addition, by Theorem 4.1 the equation in (12) is UHR stable.

\section{Conclusion}

Our proposed multiterm BVP has been successfully investigated in this work via three theorems: The Darbo's fixed point theorem (DFPT), the Kuratowski measure of noncompactness (KMNC), and the Ulam-Hyers-Rassias stability (UHR) to prove the existence and stability of solutions for our proposed BVP. A numerical example is given at the end to support and validate the potentiality of all our obtained results. As a result of our investigation into this particular research subject, our results are new and novel. Furthermore, with the support of our new results in this work, further research works can be investigated on this open research subject. Our proposed BVP can be possibly extended to other fractional models. 
Funding

Not applicable.

\section{Availability of data and materials}

Data sharing not applicable to this paper as no datasets were generated or analyzed during the current study.

\section{Ethics approval and consent to participate}

Not applicable.

\section{Competing interests}

The authors declare that they have no competing interests.

\section{Consent for publication}

Not applicable.

\section{Authors' contributions}

The authors declare that the study was realized in collaboration with equal responsibility. All authors read and approved the final manuscript.

\section{Author details}

'Laboratory ACEDP, Djillali Liabes University, Sidi Bel Abbès, Algeria. ${ }^{2}$ Department of Mathematics, Cankaya University, Ankara, Turkey. ${ }^{3}$ Institute of Space Sciences, Magurele-Bucharest, Romania. ${ }^{4}$ Department of Economic Sciences, University of Tiaret, Tiaret, Algeria. ${ }^{5}$ Department of Computer Engineering, Biruni University, Istanbul, Turkey. ${ }^{6}$ Science Faculty, Department of Mathematics, Firat University, 23119 Elazig, Turkey. ${ }^{7}$ Department of Medical Research, China Medical University Hospital, China Medical University, Taichung, Taiwan.

\section{Publisher's Note}

Springer Nature remains neutral with regard to jurisdictional claims in published maps and institutional affiliations.

\section{Received: 23 March 2021 Accepted: 8 July 2021 Published online: 03 August 2021}

\section{References}

1. Gomez-Aguilar, J.F.: Analytical and numerical solutions of nonlinear alcoholism model via variable-order fractional differential equations. Physica A 494, 52-57 (2018)

2. Sun, H., Chen, W., Wei, H., Chen, Y.: A comparative study of constant-order and variable-order fractional models in characterizing memory property of systems. Eur. Phys. J. Spec. Top. 193, 185-192 (2011)

3. Tavares, D., Almeida, R., Torres, D.F.M.: Caputo derivatives of fractional variable order numerical approximations. Commun. Nonlinear Sci. Numer. Simul. 35, 69-87 (2016)

4. da Vanterler, C., Sousa, J., Capelas de Oliverira, E.: Two new fractional derivatives of variable order with non-singular kernel and fractional differential equation. Comput. Appl. Math. 37, 5375-5394 (2018)

5. Yang, J., Yao, H., Wu, B.: An efficient numerical method for variable order fractional functional differential equation. Appl. Math. Lett. 76, 221-226 (2018)

6. Alzabut, J., Selvam, A., Dhineshbabu, R., Kaabar, M.K.A.: The existence, uniqueness, and stability analysis of the discrete fractional three-point boundary value problem for the elastic beam equation. Symmetry 13(5), 1-18 (2021)

7. Matar, M.M., Abbas, M.I., Alzabut, J., Kaabar, M.K.A., Etemad, S., Rezapour, Sh.: Investigation of the p-Laplacian nonperiodic nonlinear boundary value problem via generalized Caputo fractional derivatives. Adv. Differ. Equ. 2021 $68(2021)$

8. Can, N.H., Kumar, D., Viet, T.V., Nguyen, A.T.: On time fractional pseudo-parabolic equations with nonlocal in time condition. Math. Methods Appl. Sci. 1(19) (2021)

9. Liua, J.G., Yang, X.J., Feng, Y.Y., Cui, P.: On group analysis of the time fractional extended (2+1)-dimensional Zakharov-Kuznetsov equation in quantum magneto-plasmas. Math. Comput. Simul. 178, 407-421 (2020)

10. Liua, J.G., Yang, X.J., Feng, Y.Y., Cui, P., Geng, L.L.: On integrability of the higher dimensional time fractional KdV-type equation. J. Geom. Phys. 160, 104000 (2021)

11. Phuong, N.D., Hoan, L.V.C., Karapinar, E., Singh, J., Binh, H.D., Can, N.H.: Fractional order continuity of a time semi-linea fractional diffusion-wave system. Alex. Eng. J. 59(6), 4959-4968 (2020)

12. Singh, J., Kumar, D., Purohit, S.D., Mishra, A.M., Bohra, M.: An efficient numerical approach for fractional multidimensional diffusion equations with exponential memory. Numer. Methods Partial Differ. Equ. 37(2), 1631-1651 (2021)

13. Bouazza, Z., Etemad, S., Souid, M.S., Rezapour, S., Martínez, F., Kaabar, M.K.A.: A study on the solutions of a multiterm FBVP of variable order. J. Funct. Spaces 2021, 1-9 (2021)

14. Bai, Y., Kong, H.: Existence of solutions for nonlinear Caputo-Hadamard fractional differential equations via the method of upper and lower solutions. J. Nonlinear Sci. Appl. 10, 5744-5752 (2017)

15. Samko, S.G.: Fractional integration and differentiation of variable order. Anal. Math. 21, 213-236 (1995)

16. Samko, S.G., Boss, B.: Integration and differentiation to a variable fractional order. Integral Transforms Spec. Funct. 1 , 277-300 (1993)

17. Valerio, D., Costa, J.S.: Variable-order fractional derivatives and their numerical approximations. Signal Process. 91, 470-483 (2011)

18. Kilbas, A.A., Srivastava, H.M., Trujillo, J.J.: Theory and Applications of Fractional Differential Equations. North-Holland Mathematics Studies, vol. 204. Elsevier, Amsterdam (2006)

19. Zhang, S.: Existence of solutions for two point boundary value problems with singular differential equations of variable order. Electron. J. Differ. Equ. 245, 1 (2013) 
20. Zhang, S., Hu, L.: Unique existence result of approximate solution to initial value problem for fractional differential equation of variable order involving the derivative arguments on the half-axis. Mathematics 7(286), 1-23 (2019)

21. Zhang, S., Hu, L.: The existeness and uniqueness result of solutions to initial value problems of nonlinear diffusion equations involving with the conformable variable. Azerb. J. Math. 9(1), 22-45 (2019)

22. Zhang, S., Sun, S., Hu, L.: Approximate solutions to initial value problem for differential equation of variable order. J. Fract. Calc. Appl. 9(2), 93-112 (2018)

23. Jiahui, A., Pengyu, C.: Uniqueness of solutions to initial value problem of fractional differential equations of variable-order. Dyn. Syst. Appl. 28(3), 607-623 (2019)

24. Zhang, S.: The uniqueness result of solutions to initial value problems of differential equations of variable-order. Rev. R. Acad. Cienc. Exactas Fís. Nat., Ser. A Mat. 112, 407-423 (2018)

25. Zhang, S., Hu, L.: The existence of solutions and generalized Lyapunov-type inequalities to boundary value problems of differential equations of variable order. AIMS Math. 5(4), 2923-2943 (2020)

26. Banas̀, J., Goebel, K.: Measures of Noncompactness in Banach Spaces. Dekker, New York (1980)

27. Banas̀, J., Olszowy, L.: Measures of noncompactness related to monotonicity. Comment. Math. Prace Mat. 41, 13-23 (2001)

28. Guo, D.J., Lakshmikantham, V., Liu, X.: Nonlinear Integral Equations in Abstract Spaces. Kluwer Academic, Dordrecht (1996)

29. Rus, I.A.: Ulam stabilities of ordinary differential equations in a Banach space. Carpath. J. Math. 26, 103-107 (2010)

30. Benchohra, M., Bouriah, S., Lazreg, J.E., Nieto, J.J.: Nonlinear implicit Hadamard's fractional differential equations with delay in Banach space. Acta Univ. Palacki. Olomuc., Fac. Rerum Nat., Math. 55(1), 15-26 (2016)

\section{Submit your manuscript to a SpringerOpen ${ }^{\circ}$ journal and benefit from:}

- Convenient online submission

- Rigorous peer review

Open access: articles freely available online

High visibility within the field

Retaining the copyright to your article

Submit your next manuscript at $\boldsymbol{~ s p r i n g e r o p e n . c o m ~}$ 